\title{
Improved predictions of nuclear reaction rates for astrophysics applications with the TALYS reaction code
}

\author{
S. Goriely ${ }^{1, a}$, S. Hilaire ${ }^{2}$, and A.J. Koning ${ }^{3}$ \\ 1 Institut d'Astronomie et d'Astrophysique, Université Libre de Bruxelles, Campus de la Plaine CP 226, 1050 Brussels, Belgium \\ 2 CEA/DAM Île-de-France, DPTA/Service de Physique Nucléaire, BP. 12, 91680 Bruyères-le-Châtel, France \\ ${ }^{3}$ Nuclear Research and Consultancy Group, P.O. Box 25, 1755 ZG Petten, The Netherlands
}

\begin{abstract}
Nuclear reaction rates for astrophysics applications are traditionally determined on the basis of HauserFeshbach reaction codes, like MOST. These codes use simplified schemes to calculate the capture reaction cross section on a given target nucleus, not only in its ground state but also on the different thermally populated states of the stellar plasma at a given temperature. Such schemes include a number of approximations that have never been tested, such as an approximate width fluctuation correction, the neglect of delayed particle emission during the electromagnetic decay cascade or the absence of the pre-equilibrium contribution at increasing incident energies. New developments have been brought to the reaction code TALYS to estimate the Maxwellian-averaged reaction rates of astrophysics relevance. These new developments give us the possibility to calculate with an improved accuracy the reaction cross sections and the corresponding astrophysics rates. The TALYS predictions for the thermonuclear rates of astrophysics relevance are presented and compared with those obtained with the MOST code on the basis of the same nuclear ingredients for nuclear structure properties, optical model potential, nuclear level densities and $\gamma$-ray strength. It is shown that, in particular, the pre-equilibrium process significanly influences the astrophysics rates of exotic neutron-rich nuclei. The reciprocity theorem traditionally used in astrophysics to determine photorates is also shown no to be valid for exotic nuclei. The predictions obtained with different nuclear inputs are also analyzed to provide an estimate of the theoretical uncertainties still affecting the reaction rate prediction far away from the experimentally known regions.
\end{abstract}

\section{Introduction}

Most of the nuclear astrophysics calculations make use of nuclear reaction rates evaluated within the Hauser-Feshbach (HF) statistical model [1,2]. It relies on the fundamental assumption (Bohr hypothesis) that the capture process takes place through the intermediary production of a compound system that can reach a state of thermodynamic equilibrium. This compound system is then classically referred to as the compound nucleus $(\mathrm{CN})$. The formation of a $\mathrm{CN}$ is usually justified if its level density at the excitation energy corresponding to the projectile incident energy is large enough. In this framework, the reaction $I^{\mu}+j \rightarrow L+k$ of capture of a nucleon or $\alpha$-particle $j$ on target $I$ in its state $\mu$ leaving the residual nucleus $L$ and particle or photon $k$ has a cross section at centreof-mass energy $E$ given by

$$
\sigma_{j k}^{\mu}(E)=\pi \lambda_{j}^{2} \frac{1}{\left(2 J_{I}^{\mu}+1\right)\left(2 J_{j}+1\right)} \sum_{J^{\pi}}(2 J+1) \frac{T_{j}^{\mu}\left(J^{\pi}\right) T_{k}\left(J^{\pi}\right)}{T_{\mathrm{tot}}\left(J^{\pi}\right)},
$$

where $J_{I}$ and $J_{j}$ are the target and projectile spins, and $T_{j}\left(J^{\pi}\right)$ is the transmission coefficient measuring the probability for forming the $\mathrm{CN}$ (i.e., the compound system assumed in the HF model to have reached a state of thermodynamic equilibrium) in its state $J^{\pi}$ obtained from all possible combinations of the orbital and channel spins. Similarly, $T_{k}\left(J^{\pi}\right)=\sum_{v} T_{k}^{v}\left(J^{\pi}\right)$ is the transmission coefficient for the decay of the compound nuclear state into the pair $L+k$, all states $v$ of $L$ which can be populated

\footnotetext{
${ }^{a}$ Presenting author, e-mail: sgoriely@astro.ulb.ac.be
}

in the reaction being taken into account. $T_{\text {tot }}\left(J^{\pi}\right)=\sum_{i, \lambda} T_{i}^{\lambda}\left(J^{\pi}\right)$ corresponds to the total transmission coefficient for the decay of the compound state $J^{\pi}$ into any combination $i$ of nucleus and particle which can be formed from all its possible decay modes $\lambda$ (including $I+j$ and $L+k$ ). It has to be noted that equation (1) is valid only if the formation and decay of the $\mathrm{CN}$ are totally independent. This so-called Bohr hypothesis may not be fully satisfied, particularly in cases where a few strongly and many weakly absorbing channels are mixed. As an example, equation (1) is known to be invalid when applied to the elastic channel, since in that case the transmission coefficients for the entrance and exit channels are identical, and hence correlated. This causes an elastic enhancement. To account for these deviations, width fluctuation corrections can be introduced in the HF formalism by different approximate expressions [3].

A thermodynamic equilibrium holds locally to a very good approximation in stellar interiors. Consequently, the energies of both the targets and projectiles, as well as their relative energies $E$, obey Maxwell-Boltzmann distributions corresponding to the temperature $T$ at that location (or a backbody Planck spectrum for photons). In such conditions, the astrophysical rate is obtained by integrating the cross section given by equation (1) over a Maxwell-Boltzmann distribution of energies $E$ at the given temperature $T$. In addition, in hot astrophysical plasmas, a target nucleus exists in its ground as well as excited states. In a thermodynamic equilibrium situation, the relative populations of the various levels of nucleus $I^{\mu}$ with excitation energies $\varepsilon_{I}^{\mu}$ obey a Maxwell-Boltzmann distribution. The effective stellar rate of $I+j \rightarrow L+k$ per pair 
of particles in the entrance channel at temperature $T$ taking due account of the contributions of the various target excited states is finally expressed in a classical notation as

$$
\begin{aligned}
N_{A}\langle\sigma v\rangle_{j k}^{*}(T)= & \left(\frac{8}{\pi m}\right)^{1 / 2} \frac{N_{A}}{(k T)^{3 / 2} G(T)} \int_{0}^{\infty} \sum_{\mu} \frac{\left(2 J_{I}^{\mu}+1\right)}{\left(2 J_{I}^{0}+1\right)} \\
& \times \sigma_{j k}^{\mu}(E) E \exp \left(-\frac{E+\varepsilon_{I}^{\mu}}{k T}\right) d E
\end{aligned}
$$

where $k$ is the Boltzmann constant, $m$ the reduced mass of the $I^{0}+j$ system, $N_{A}$ the Avogadro number, and

$$
G(T)=\sum_{\mu}\left(2 J_{I}^{\mu}+1\right) /\left(2 J_{I}^{0}+1\right) \exp \left(-\varepsilon_{I}^{\mu} / k T\right)
$$

the $T$-dependent normalized partition function. Reverse reactions can also be estimated making use of the reciprocity theorem [2]. In particular, the stellar photodissociation rates are classically derived from the radiative capture rates by

$$
\begin{aligned}
\lambda_{(\gamma, j)}^{*}(T)= & \frac{\left(2 J_{I}+1\right)\left(2 J_{j}+1\right)}{\left(2 J_{L}+1\right)} \frac{G_{I}(T)}{G_{L}(T)}\left(\frac{A_{I} A_{j}}{A_{L}}\right)^{3 / 2}\left(\frac{k T}{2 \pi \hbar^{2} N_{A}}\right)^{3 / 2} \\
& \times N_{A}\langle\sigma v\rangle_{(j, \gamma)}^{*} \mathrm{e}^{-Q_{j \gamma} / k T}
\end{aligned}
$$

where $Q_{j \gamma}$ is the Q-value of the $I^{0}(j, \gamma) L^{0}$ capture. Note that, in stellar conditions, the reaction rates for targets in thermal equilibrium are usually believed to obey reciprocity since the forward and reverse channels are symmetrical, in contrast to the situation which would be encountered for targets in their ground states only [2]. The total stellar photodissociation rate can also be determined directly from

$$
\lambda_{(\gamma, j)}^{*}(T)=\frac{\sum_{\mu}\left(2 J^{\mu}+1\right) \lambda_{(\gamma, j)}^{\mu}(T) \exp \left(-\varepsilon^{\mu} / k T\right)}{\sum_{\mu}\left(2 J^{\mu}+1\right) \exp \left(-\varepsilon^{\mu} / k T\right)},
$$

where the photodissociation rate $\lambda_{(\gamma, j)}^{\mu}$ of state $\mu$ with excitation energy $\varepsilon^{\mu}$ is given by

$$
\lambda_{(\gamma, j)}^{\mu}(T)=\int_{0}^{\infty} c n_{\gamma}(E, T) \sigma_{(\gamma, j)}^{\mu}(E) d E,
$$

where $c$ is the speed of light, $\sigma_{(\gamma, j)}^{\mu}(E)$ the photodisintegration cross section at energy $E$, and $n_{\gamma}$ the stellar $\gamma$-ray distribution well described by the back-body Planck spectrum at the given temperature $T$.

The uncertainties involved in any HF cross section calculation are of two origins. The first one is related to the reaction mechanism, i.e., the model of formation and de-excitation of the $\mathrm{CN}$ itself. In particular, at increasing energies or for nuclei for which the $\mathrm{CN}$ does not have time to reach a thermodynamic equilibrium, direct or pre-equilibrium processes might become significant. The pre-equilibrium emission can take place after the first stage of the reaction but long before statistical equilibrium of the $\mathrm{CN}$ is achieved. The incident particle stepby-step creates more complex states in the compound system and gradually loses its memory of the initial energy and direction. Pre-equilibrium processes can cover a sizable part of the reaction cross section for intermediate energies (typically more than a few $\mathrm{MeV}$ ), but have always been neglected to estimate the reaction rates for astrophysics applications. Such effects are studied in section 2. More systematically, the predictions obtained with the TALYS [4] and MOST [5,6] codes are compared in section 2. In the most extreme case, i.e., for light or systems for which no resonant states are available (like neutron-rich nuclei), the direct capture might dominate the reaction mechanism. This process has been studied in [7] and will not be further discussed here.

Another type of uncertainties come from the evaluation of the nuclear quantities necessary for the calculation of the transmission coefficients entering equations (1)-(2), i.e., the ground and excited state properties (masses, deformations, matter densities, excited state energies, spins and parities...), nuclear level densities, $\gamma$-ray strength, optical model potential and fission properties. When not available experimentally, this information has to be obtained from nuclear models. Ideally, these various ingredients should be derived from global, universal and microscopic models [5,6]. Over the last decades, much progress has been made in the development of global microscopic models. For applications, in particular of astrophysics nature, where nuclei far from stability are involved, the use of microscopic models has clearly to be preferred. This is even more so as new generations of such models are started to be developed which can compete with more phenomenological highly-parametrized models in the reproduction of experimental data [5,6,8-10]. Nuclear ingredients from global microscopic models have been included in TALYS and are shown to predict quite different astrophysical rates (sect. 3).

\section{Comparison between MOST and TALYS}

All the details about the code MOST can be found in ref. [5,6] and about TALYS in ref. [4]. The present section aims at considering the two different codes in their prediction of the astrophysics rates, using the same nuclear input models to describe the ground state properties [8], the nuclear level density [9], the $\gamma$-ray strength [10], the optical model potential [11]. With such inputs, both codes reproduce globally the experimental neutron, proton or photo-rates with the same accuracy.

Figure 1 illustrates the deviation that could exist between MOST and TALYS predictions of the radiative neutron capture rates for all nuclei with $16 \leq Z \leq 83$ between the neutron and the proton drip lines at the temperature of $T_{9}=1$ (where $T_{9}$ is the expressed in $10^{9} \mathrm{~K}$ ).

As far as the calculation of the maxwellian-averaged reaction rates of astrophysics interest is concerned, the TALYS code present some clear added values with respect to the codes developed for astrophysics applications (like MOST), such as the inclusion of pre-equilibrium reaction mechanism, the detailed competition between all open channels, in particular, delayed particle emission (which are not taken consistently into account in astrophysics codes), the inclusion of multi-particle emission (totally neglected in astrophysics codes), the inclusion of detailed width fluctuation corrections $[12,13]$ in comparison with the approximation of ref. [14] used 


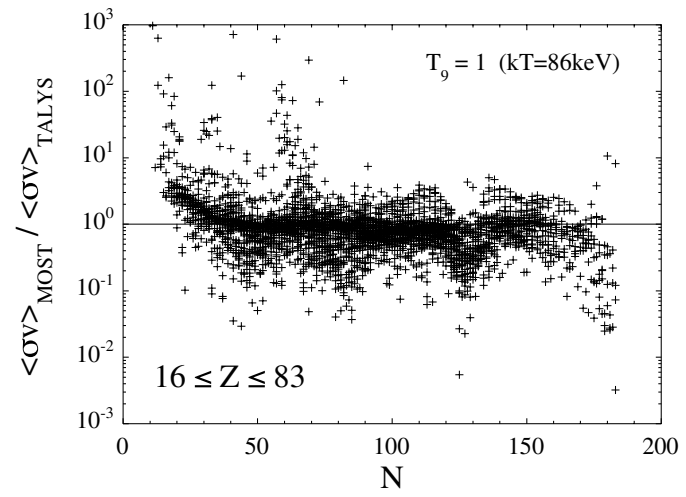

Fig. 1. Ratio of MOST to TALYS radiative neutron capture rates for all nuclei with $16 \leq Z \leq 83$ at $T_{9}=1$ as a function of the neutron number $N$.

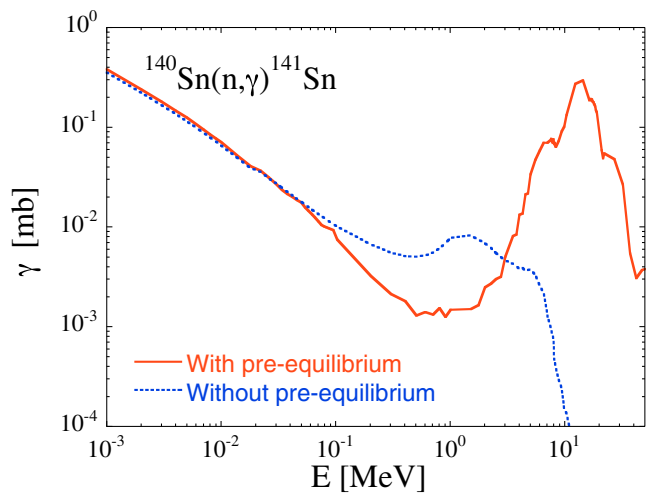

Fig. 2. Radiative neutron capture cross section for ${ }^{140} \mathrm{Sn}$ with and without including the pre-equilibrium processes as a function of energy.

in astrophysics codes, the inclusion of parity-dependent level densities, the inclusion of coupled channel description for deformed nuclei, and the coherent inclusion of fission channel. Different prescriptions are also used when renormalizing nuclear models on available experimental data, such nuclear level densities on s-wave spacings or E1 resonance strength on photoabsorption data. When considering astrophysics applications, i.e., thermonuclear reaction rates at astrophysically relevant temperatures, most of the approximations made in a code like MOST can be justified, since for neutron captures essentially incident energies of a few $\mathrm{keV}$ to $1 \mathrm{MeV}$ are concerned, and for incident charged particles, only energies below or close to the coulomb barrier come into play. Most of these approximations therefore do not affect the predicted rates by more than a few tens of a percent.

However, among the approximations made in astrophysics codes like MOST, one turn out to have a significant impact on the rate; it concerns the contribution of the pre-equilibrium process. The pre-equilibrium process is known to play an important role for stable nuclei at incident energies of a few $\mathrm{MeV}$, which hardly affect the Maxwellian-averaged reaction rates of astrophysics relevance. However, if we consider exotic neutron-rich nuclei with low neutron separation energies, the low-level density makes it difficult for the nucleus to become fully equilibrated. In this case, the pre-equilibrium process

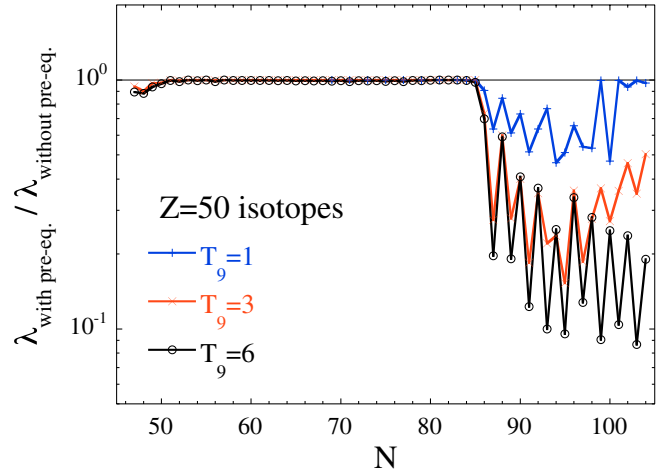

Fig. 3. Ratio of the photoneutron rates obtained with and without including the pre-equilibrium process for all $\mathrm{Sn}$ isotopes at three different temperatures.

starts to affect the neutron channel already at a few hundred $\mathrm{keV}$. An example is shown in figure 2, where already at $100 \mathrm{keV}$ the pre-equilibrium process plays an important role. Note that the pre-equilibrium model adopted here corresponds to the two-component exciton model [15]. The pre-quilibrium process is found to affect not only the nucleon or $\alpha$-particle capture rates but also the photoneutron rates of neutron-rich nuclei, as illustrated in figure 3 . Since the pre-equilibrium component contributes at increasing energies, the higher the temperature the larger the impact on the astrophysics rates, as seen in figure 3 .

When the pre-equilibrium process influences the stellar reaction cross section, it is intuitively clear that the emitted particle keeps some memory of the incident energy and direction, so the hypothesis of the fully equilibrated $\mathrm{CN}$ is not valid, and consequently the reciprocity theorem [2] relating the direct and inverse reaction rates (see eq. (4)) may not apply anymore. Other effects like delayed particle emission or width fluctuation correction reflecting the correlation between the incoming and outgoing channel can also be expected to invalidate the reciprocity theorem. To show to what extent the reciprocity theorem is valid, we compare in figure 4 the photoneutron rate estimated directly with the code TALYS (eq. (5)) with the one derived from eq. (4). Deviations up to a factor of 10 are observed for neutron-rich nuclei. This decrease of the photorates with respect to the value traditionally derived from the reciprocity theorem can in fluence the r-process nucleosynthesis. Indeed, the $(n, \gamma)-(\gamma, \mathrm{n})$ equilibrium, even if reached, is not function of the neutron separation energy anymore, but well of the reaction rates. The resulting decrease of the photodisintegration rate favours the nuclear flow into the neutron-rich region.

\section{Nuclear inputs to the TALYS code}

The nuclear ingredients of relevance to the Hauser-Feshbach calculations are taken from the latest experimental compilations when available, or otherwise, from theoretical estimates based either on classical macroscopic theories or preferentially on microscopic models. Microscopic models are believed to be of higher reliability due to their sound 


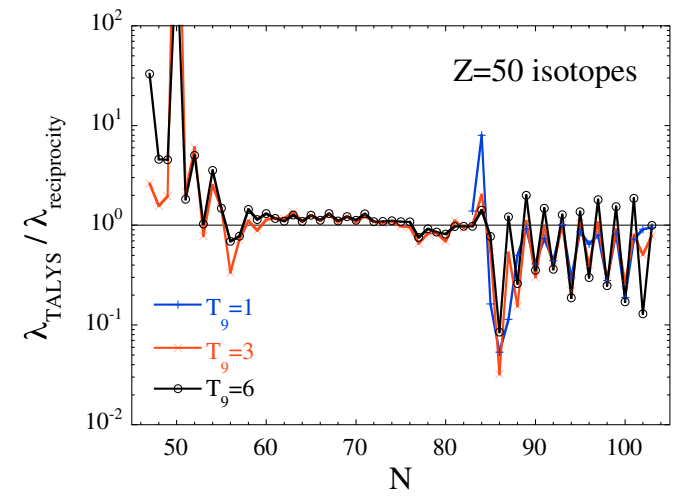

Fig. 4. Ratio of the photoneutron rates obtained with and without including the pre-equilibrium process for all $\mathrm{Sn}$ isotopes at three different temperatures.

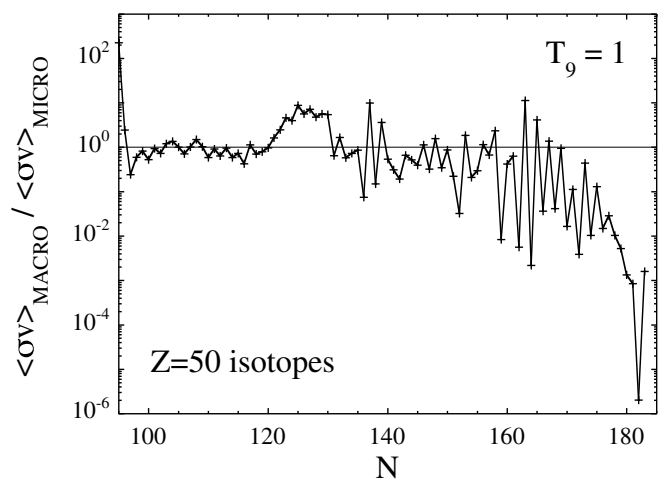

Fig. 5. Ratio of the photoneutron rate obtained with the macroscopic nuclear ingredients to the one derived with the microscopic input, as defined in the text, for all $\mathrm{Sn}$ isotopes at $T_{9}=1$.

physical description and therefore better suited for extrapolation far away from experimentally known regions of the nuclear chart. Such microscopic models have been shown to predict experimental data with the same degree of accuracy as the more phenomenological models traditionally used for practical applications. Microscopic models for ground state properties, nuclear level densities, $\gamma$-ray strength function and fission properties are included in TALYS, as already described in section 2. Additional models are also available in TALYS. In particular, we can define a macroscopic input set based on the FRDM mass model [16], the back-shilfted Fermi gas model of nuclear level densities [17] and the Lorentzian-type $\gamma$-ray strength [18]. Both the so-called microscopic and macroscopic input provide similar prediction for the reaction rates on stable nuclei. However, when dealing with exotic neutron-rich nuclei, deviations of factor of 10-100 can be observed (fig. 5), especially for even $\mathrm{N}$ isotopes for which the neutron separation energy is relatively lower. Note that both calculations include the same optical potential [11]. It can however be shown [19] that substantial uncertainties still affect the isovector part of the imaginary potential that could decrease significantly the neutron capture rates of exotic neutron-rich nuclei. This type of uncertainties have not been included in figure 5 .

\section{Conclusion}

New developments have been brought to the reaction code TALYS to estimate the Maxwellian-averaged reaction rates of astrophysics relevance. In addition, microscopic nuclear models for ground state properties, nuclear level densities and $\gamma$-ray strength function have been included. These new developments give us the possibility to estimate with an improved accuracy the reaction cross sections and the corresponding astrophysics rates. The TALYS predictions for the thermonuclear rates of astrophysics relevance are found to be relatively similar to the more traditional codes for stable nuclei, but major differences are obtained when dealing with exotic neutron-rich nuclei. In particular, the pre-equilibrium process is found to play a major role at low energies for neutron-rich nuclei. The reciprocity theorem traditionally used in astrophysics to estimate photorates is also shown not to be strictly valid for exotic nuclei.

S.G. is F.N.R.S. senior research assistant.

\section{References}

1. W. Hauser, H. Feshbach, Phys. Rev. 87, 366 (1952).

2. J.A. Holmes, S.E. Woosley, W.A. Fowler, B.A. Zimmerman, At. Data Nucl. Data Tables 18, 306 (1976).

3. S. Hilaire, C. Lagrange, A.J. Koning, Ann. Phys. 306, 209 (2003).

4. A.J. Koning, S. Hilaire, M. Duijvestijn (these proceedings).

5. M. Aikawa, M. Arnould, S. Goriely et al., Astron. Astrophys. 441, 1195 (2005).

6. M. Arnould, S. Goriely, Nucl. Phys. A 777, 157c (2006).

7. S. Goriely, Astron. Astrophys. 325, 414 (1997).

8. S. Goriely, J.M. Pearson, M. Samyn, Nucl. Phys. A 773, 279 (2006).

9. S. Hilaire, S. Goriely, Nucl. Phys. A 779, 63 (2006).

10. S. Goriely, E. Khan, Nucl. Phys. A 706, 217 (2002).

11. A.J. Koning, J.P. Delaroche, Nucl. Phys. A 713, 231 (2003).

12. P.A. Moldauer, Nucl. Phys. A 344, 185 (1980).

13. J.J.M. Verbaarschot, H.A. Weidenmüller, M.R. Zirnbauer, Phys. Rep. 129, 367 (1985).

14. H.M. Hofmann, T. Mertelmeier, M. Herman, J.W. Tepel, Zeit. Phys. A 297, 153 (1980).

15. A.J. Koning, M.C. Duijvestijn, Nucl. Phys. A 744, 15 (2004).

16. P. Möller, J.R. Nix, W.D. Myer, W.J. Swiatecki, At. Data Nucl. Data Tables 59, 185 (1995).

17. Handbook for calculations of nuclear reaction data, RIPL-2 (IAEA-Tecdoc-1506, 2006).

18. J. Kopecky, M. Uhl, Phys. Rev. C 42, 1941 (1990).

19. S. Goriely, J.P. Delaroche, Phys. Lett. B (2007) (submitted). 